\title{
ROLE OF SEQUENCING DNA IN THE HUMAN PAPILLOMAVIRUS TYPING. DIAGNOSTIC AND PROGNOSTIC PERSPECTIVES.
}

\author{
Leo G.', Pisano' M.', Ciannamea B. ${ }^{2}$, Pitotti E.', \\ Castellano S.', Vergara D.', Megha M.', Moschettini G. ${ }^{3}$, \\ Mighali P. ${ }^{3}$ and Maffia M. ${ }^{3}$ \\ 'U.O. Molecular Biology and Experimental Oncology \\ (P. Oncologico "V. Fazzi"- AUSL LE/I), \\ 2U.O. Microbiology and Virology (AUSL LE/I), \\ ${ }^{3}$ Dept. of Biological and Environmental Sciences and Technologies \\ - University of Lecce (Italy).
}

Introduction. Various methods have been used to identify HPV genotypes after amplification with general and consensus primer. In this preliminary study, we want to evaluate the performance of one of these assays: sequence analysis.

Methods. We have compared the results of HPV detection and typing from 20 cervical samples assayed by two methods: L1 (My 09/11) and E6/E7 (pV-1M, 31B, 2R) consensus PCR followed by cycle sequencing and subsequent BLAST searching and L1 (My 09/11) consensus PCR followed by commercial reverse hybridization L1 probes assay.

Results. Our results indicate that discordant cases (probe vs sequence) were observed in four samples, out of twenty L1 sequences studied. In two of these, the probe assay identified more than one HPV types while sequence analysis detected only one $(16+31$ vs $16 ; 16+31$ vs 16$)$. In other two samples, defined as $6+16$ and 31 on the basis of probes assay, sequence analysis detected a multiple infection of $6+53$ and a single infection of 16 respectively. In addition, cycle sequencing detected three HPV types not included in the commercial assay. Among the twenty HPV L1 positives, only three cases were not recognized by analysis of E6/E7 fragment; these several discordant results are due to limit of these primers to detect some types of HPV. On the contrary, one sample, negative by analysis of L1 region, was identified as 16 genotype by E6/E7 primers (integration into the host chromosomal DNA?).

The sequence of L1 region of all HPV 16 positive samples showed also a perfect homology $(100 \%)$ with the sequence of HPV 16 East Asian type in Gen Bank.

Conclusions. Sequence analysis provides a new approach in the detection of different HPVs and has several advantages. These advantages include increased precision in characterization of single HPVs better than probes assay, and unique ability to detect mutations and identify new HPV types. 